\title{
A modelling study of the cardiovascular response to exercise in patients with mitral stenosis
}

\author{
E. Magosso \& M. Ursino \\ Department of Electronics, Computer Science, and Systems, \\ University Of Bologna, Italy
}

\begin{abstract}
The objective of the present study was to quantitatively investigate the effect of mitral valve stenosis on the hemodynamics, both at rest and during exercise. The analysis was performed by means of a mathematical model. The model includes the pulsating heart, the pulmonary and systemic circulation, and the main mechanisms involved in exercise response: the ventilatory adjustments to exercise, the muscle and respiratory pump, the local metabolic vasodilation in active muscles, the central motor command, the arterial baroreflex, and the lung inflation reflex. With basal parameter values, the model is able to reproduce the cardiovascular response measured in healthy human subjects to different levels of aerobic exercise. Simulation of moderate mitral stenosis conditions shows that the pathological status at rest is partially masked by the action of the baroreflex. However, the depressed cardiac reserve is clearly revealed even at mild exercise: the stenosed heart cannot increase its output to a normal extent, seriously reducing patient's exercise tolerance. Moreover, pulmonary venous pressure increases up to a high-risk level of pulmonary edema. The model allows a quantitative interpretation of the complex interaction between regulatory mechanisms and cardiocirculatory parameters during exercise.
\end{abstract}

\section{Introduction}

Examination of the hemodynamic response to exercise is commonly used in the clinical practice, as a test for the diagnosis of cardiovascular diseases. The main idea behind these tests is that, thanks to the action of regulatory mechanisms, a 
pathological subject may have quite normal cardiovascular quantities at rest (i.e., almost normal levels of systemic arterial pressure, cardiac output and peripheral blood flow) despite the disease state. During stress conditions, however, the regulatory mechanisms are no longer able to compensate for the increased need in cardiac output and local blood flow, thus resulting in poor cardiovascular performance compared with a normal healthy subject.

A quantitative description of this problem requires the simultaneous comprehension of various physiological aspects, namely: i) A description of the cardiovascular circulation (both systemic and pulmonary) as well as of heart functioning, and of its possible alterations in the pathological states. ii) Analysis of the cardiovascular regulatory actions which can compensate for the alterations at rest. iii) An investigation of the regulation mechanisms which participate in the exercise response to warrant adequate cardiac output and oxygen delivery, and of their interaction with the other mechanisms.

Mathematical modeling and computer simulation techniques may be of great value to reach a deeper understanding of this problem, and to clarify the complex interrelationships among the different mechanisms involved. Despite its evident clinical importance, however, an accurate and physiologically sounded quantitative analysis of exercise hemodynamics, and of its alterations during cardiovascular diseases is still lacking.

In recent years we developed a comprehensive mathematical model of shortterm cardiovascular regulation, which incorporates a large body of present physiological knowledge about short-term cardiovascular control. The model, with reliable parameter values, is able to simulate cardiovascular adjustments to a variety of perturbations, such as hemorrhage, hypoxia, asphyxia [1-4].

Aim of the present work is to further extend the former model, including also a description of the regulatory mechanisms responsible for the hemodynamic adjustments to aerobic dynamic exercise. The model is then used to compare the cardiovascular response to exercise in a normal subject and in a subject with mitral stenosis. This particular disease has been chosen since its effect on hemodynamics at rest can be simulated quite well and, moreover, patients with mitral stenosis have a limited exercise tolerance $[5,6]$.

The paper is structured as follows: first, the main aspects of the model are presented, laying particular emphasis on the new equations. The original aspects are then validated by comparing model's response at various levels of aerobic dynamic exercise with data taken from the literature. Finally, the model is used to simulate the hemodynamics in a patient with severe mitral stenosis both at rest and in response to dynamic exercise. The results show that regulatory mechanisms warrant acceptable values of arterial pressure and cardiac output at rest, despite the presence of mitral stenosis, but the pathological status becomes dramatically evident even at moderate exercise levels.

\section{Model description}

The model comprehends the pulsating heart, the pulmonary and systemic circulation, the mechanical effects of respiration and muscular contractions on 
venous return (muscle pump and respiratory pump), and the action of several active control mechanisms. The only input for the model is the relative intensity of aerobic exercise (indicated by $I$ in the following), normalized with respect to the individual maximum oxygen uptake. $I$ may range between 0 and $1: I$ equals to 0 corresponds to resting conditions, while $I$ equals to 1 represents the threshold between aerobic and anaerobic exercise.

\subsection{The cardiovascular system}

The model of the pulsating heart (both left and right) is the same as in a previous study [1], where an accurate description can be found. Briefly, each atrium is described as a linear capacity, characterized by constant value of compliance and unstressed volume, i.e., the contractile activity of the atrium is neglected. Blood passes from the atrium to the ventricle through the atrio-ventricular valve, mimicked as the series arrangement of an ideal diode and a constant resistance. The contractile activity of the ventricle is simulated by means of a variable elastance model. Shifting from the end-diastolic to the end-systolic pressurevolume curve is governed by a pulsating excitation function with period equal to heart period. Finally, blood leaves the ventricle through the aortic (pulmonary) valve, simulated by means of an ideal diode.

The vascular system contains the pulmonary circulation (consisting of the series arrangement of the large arteries, peripheral and venous circulation), and the systemic circulation. The latter includes the large arteries and distinguishes between the peripheral and venous circulation of six parallel vascular districts: brain, heart, active skeletal muscles (corresponding to leg skeletal muscles), resting skeletal muscles, the splanchnic circulation, and the remaining extrasplanchnic vascular beds. This distinction is necessary since regulatory mechanisms exert a different action on each of these districts. Finally, a further compartment mimics the thoracic veins returning blood to the right heart.

Hemodynamics in each compartment is described by means of a resistive term, which accounts for the pressure energy losses, and a capacitive term, which describes the blood volume stored in the compartment at a given transmural pressure. Inertial effects have been included only in the large artery compartments, where blood acceleration is significant. We used a linear pressure-volume curve (characterized by compliance and unstressed volume) in all the compartments, except in the active muscle veins, where a more complex description has been adopted in order to reproduce the muscle pump effects. Muscular contractions, indeed, may induce a negative transmural pressure at the veins, causing them to collapse and resulting in a large expulsion of blood volume (muscle pump). To describe this phenomenon, we assumed that extravascular pressure at active muscle veins (i.e., intramuscular pressure) oscillates rhythmically between zero and a positive value during exercise. Moreover, pressure-volume characteristic of the same compartment has been given two different expressions, depending on whether transmural pressure is positive or negative. At positive values of transmural pressure, the classical linear pressure-volume characteristic has been adopted (as in all other 
compartments), while at negative values, a non linear relationship for collapsing tubes was used [7]. Hence, the following equation holds:

$$
P_{a m v}-P_{i m}= \begin{cases}\frac{1}{C_{a m v}} \cdot\left(V_{a m v}-V_{u, a m \nu}\right), & V_{a m v}>V_{u, a m v} \\ P_{0} \cdot\left[1-\left(\frac{V_{a m v}}{V_{u, a m \nu}}\right)^{-\frac{3}{2}}\right], & V_{a m v}<V_{u, a m v}\end{cases}
$$

where $P_{a m v}$ is the pressure inside the active muscle veins, and $P_{i m}$ is the intramuscular pressure. $V_{a m v}$ is the total blood volume (stressed + unstressed) in the active muscle veins, $V_{u, a m \nu}$ is the unstressed volume, $C_{a m v}$ represents the compliance of the compartment when veins are open, and finally $P_{0}$ is a constant parameter.

The mechanical effects of respiration on venous return have been simulated by using a time varying expression for intrathoracic pressure, outside the intrathoracic vascular beds (heart, pulmonary vessels, and systemic thoracic veins), and for abdominal pressure, outside splanchnic circulation. The pattern of intrathoracic and abdominal pressures depends on the depth and frequency of breathing, which both increase with exercise level.

Equations relating pressures and flows in each compartment are written by imposing the conservation of mass at the capacities and the equilibrium of forces at the inertances.

Basal values for cardiovascular parameters, as well as values for parameters of intramuscular, abdominal and intrathoracic pressure (both at rest and during exercise), have been assigned on the basis of the clinical and physiological literature, in order to reproduce the hemodynamics in a healthy human subject.

\subsection{The control system}

The control system embraces local metabolic regulation, working on active muscle and coronary vessels, and various neural mechanisms. In the description of neural mechanisms, a distinction is made between afferent pathways, efferent (sympathetic and vagal) pathways, and the response of several effectors.

The afferent pathways transport information from arterial baroreceptors, which respond to changes in systemic arterial blood pressure, and lung stretch receptors, sensitive to tidal volume variations. Both afferent pathways are described through a first-order dynamic in series with a static characteristic. Since equations are the same as used in [1,2], they are not repeated for briefness.

Efferent pathways comprehend four different kinds of fibers: sympathetic fibers to arterioles, to veins, and to heart, and the vagus. Activities in the efferent sympathetic and parasympathetic fibers are non linear functions of the weighted sum of afferent information, where the weights may be positive or negative depending on whether afferent information is excitatory or inhibitory. The 
equations describing the dependence of sympathetic and parasympathetic activities on the afferent inputs are reported in previous works [2,4].

Moreover, in the present model sympathetic and vagal activities are directly influenced, through an additive term, by the central motor command (i.e., the same command which triggers the motor movements). According to the physiological literature [6], the central command has an inhibitory effect on the vagus, while it excites the sympathetic system. The additive terms describing the central command are sigmoidal functions of exercise intensity, that remain equal to zero as long as $I$ is below a certain threshold, than rise to an upper saturation. The following equations have been used:

$$
\gamma_{i}=\frac{\gamma_{\min , i}+\gamma_{\max , i} \cdot \exp \left(\frac{I-I_{0, i}}{k_{c c, i}}\right)}{l+\exp \left(\frac{I-I_{0, i}}{k_{c c, i}}\right)}, \quad i=s p, s v, s h, v
$$

where $\gamma_{i}(i=s p, s v, s h, v)$ represent the additive terms in the sympathetic activity to the peripheral circulation, to the veins, to the heart, and in the vagal activity, respectively, $\gamma_{\min , i}$ and $\gamma_{\max , i}$ are the upper and lower saturation of the central command response, $I_{0, i}$ is the value of exercise intensity at the central point of the sigmoid, and $k_{c c, i}$ is a parameter related to the slope of the characteristic at the central point.

Cardiovascular effectors for reflex regulation include: i) the response of systemic peripheral resistances and systemic venous unstressed volumes to sympathetic fibers; ii) the response of heart contractility to cardiac sympathetic nerves; iii) and the heart period response to cardiac sympathetic nerves and the vagus. Equations describing effector response (including a static relationship, a pure delay, and a first-order dynamic) are the same as used previously $[1,2]$.

Metabolic regulation comprises a direct effect of vasodilatory metabolites, and an indirect oxygen-dependent effect mediated by tissue hypoxia. The first mechanism acts only on peripheral resistance in active muscles (where production of metabolites is conspicuous), while the second mechanism affects peripheral resistance both in active muscle and coronary vascular beds. The following equation holds for active muscle peripheral resistance:

$$
R_{a m p}=\frac{R_{a m p, n}}{\left(1+x_{a m, O_{z}}+x_{m e t}\right)}
$$

where $R_{a m p}$ is peripheral resistance in active skeletal muscle compartment, and $x_{a m, O_{2}}$ and $x_{m e t}$ are state variables, representing the effect of tissue hypoxia and of the other vasodilatory substances, respectively. $R_{a m p, n}$ is the value of active muscle peripheral resistance resulting from the neural control. The coronary peripheral resistance obeys to a similar equation, but with $x_{m e t}$ equal to zero.

The stimulus for the oxygen-dependent mechanism is assumed to be the oxygen concentration in venous blood leaving the compartment, computed by imposing a mass balance between oxygen extraction and oxygen consumption 


\section{4}

Simulations in Biomedicine $V$

rate. According to clinical data [8], muscle oxygen consumption increases linearly with $I$, while oxygen consumption in the heart increases with the average power of cardiac pump. Equations reproducing the action of this mechanism are the same as used previously $[2,4]$.

The action of vasodilatory metabolites is described, as a function of exercise intensity, through a sigmoidal static relationship and a first order dynamic. The following equation has been used for the static characteristic:

$$
x_{m e t}=\frac{x_{\text {min }}+x_{\text {max }} \cdot \exp \left(\frac{I-I_{0, \text { met }}}{k_{\text {met }}}\right)}{I+\exp \left(\frac{I-I_{0, \text { met }}}{k_{\text {met }}}\right)}
$$

where the meaning of the symbols is analogous as in eqn (2).

Finally, the model includes an empirical description of the respiratory response to exercise (both tidal volume and respiratory rate).

In the parameter assignment procedure, we tried to characterize each part of the control system separately on the basis of specific input-output experiments taken from the literature. However, parameters in the sigmoidal relationships describing the central command effects (eqn (2)) and the oxygen-independent metabolic vasodilation (eqn (4)), have been assigned to reproduce the final cardiovascular response measured at different levels of exercise in healthy individuals [8] (see Figure 1), since input-output experiments individually characterizing these mechanisms are not available in the literature.

\section{Results}

\subsection{Model validation}

Figure 1 shows the percentage changes in the mean values of the main systemic cardiovascular quantities (systemic arterial pressure, cardiac output, heart rate, systemic vascular conductance) and local quantities in exercising muscles (blood flow and oxygen consumption rate) simulated with the model and measured "in vivo" at different levels of exercise, in steady state conditions. The mean values in the model were computed, starting from the steady state pulsating waveforms (i.e., after the transient response to the perturbation was exhausted), by using the trapezoidal integration method. In vivo data are measured by Pawelczyk [8] in healthy human subjects performing two-legs cycling exercise in supine position, in the range $40-80 \%$ of maximal oxygen uptake. The agreement between model prediction and real data, ensuing from the parameter identification procedure, is good in the entire range of exercise examined. In particular, arterial pressure increases moderately with exercise intensity, despite the great rise in cardiac output. Systemic vascular conductance increases significantly, since the metabolic vasodilation in active tissues largely overcomes the moderate vasoconstriction in the other vascular beds. Heart rate exhibits a large rise, to sustain cardiac output. Finally blood flow in active muscles increases drastically (by more than 10-fold), to supply the increased metabolic requirements. 

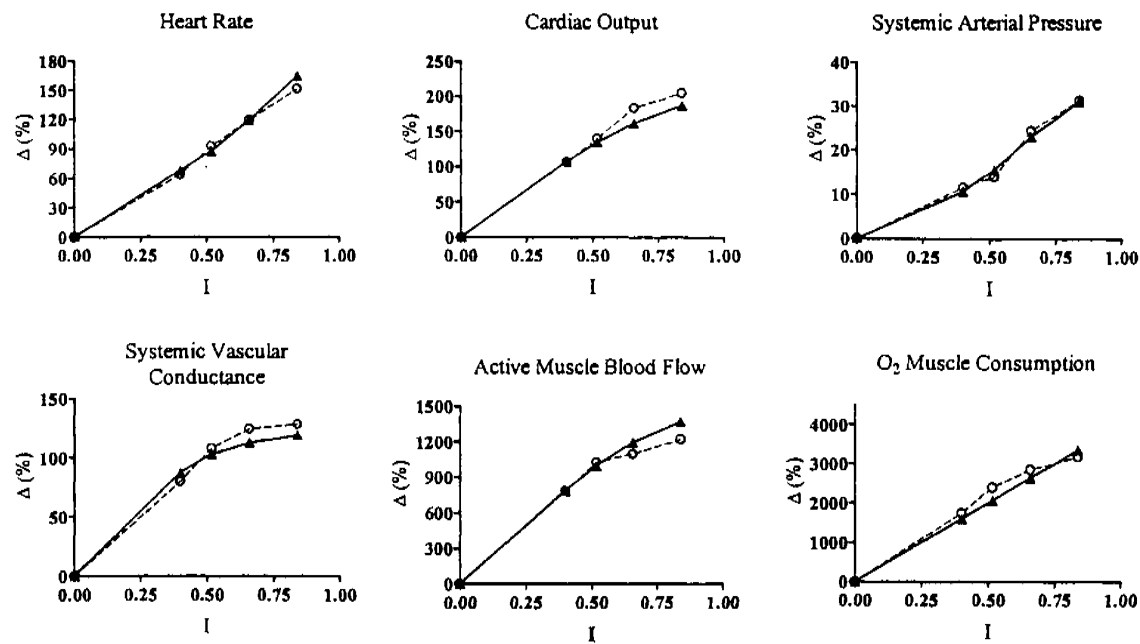

$\mathrm{O}_{2}$ Muscle Consumption

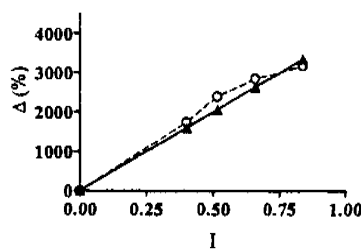

Figure 1: Percentage changes in the main cardiovascular quantities versus relative exercise intensity $(I)$ in the model and in-vivo, in steady-state conditions. 1 : model results; $O$ : clinical data measured by Pawelczyk [8] in human volunteers performing two-legs cycling exercise.

\subsection{Effect of mitral stenosis on the exercise response}

Stenosis of mitral valve has been reproduced by using a value for the resistance connecting left atrium to left ventricle about 40 fold higher then basal. This value was assigned to obtain values of left atrial pressure $(14 \mathrm{mmHg}$ vs. $6 \mathrm{mmHg}$ in the normal subject) and cardiac output $(4.3 \mathrm{l} / \mathrm{min}$ vs. $5.3 \mathrm{l} / \mathrm{min})$, in agreement with those reported in the literature for patients with moderate mitral stenosis $[5,9,10]$. Hence, the hemodynamics, both at rest and during exercise, has been simulated in the stenosed patient, and compared with that of a healthy subject.

According to Figure 2, hemodynamics of a patient with mitral stenosis at rest $(I=0)$ is characterized by a stroke volume much lower than normal, due to the decreased filling of the ventricle. As a consequence, cardiac output and arterial pressure are lower, too. Pulmonary venous pressure is raised, since the left ventricle is weaker than the right. However, it is important to note that the final steady-state condition depicted in Figure 2 at rest does not only reflect the insufficient filling of the ventricle, but also the consequent action of the regulatory mechanisms. In particular, the fall in arterial pressure activates the baroreflex control system, which produces the evident increase in heart rate, through excitation of the sympathetic system and vagal withdrawal, as well as vasoconstriction, in an effort to restore more adequate hemodynamic levels. As a consequence, cardiac output only moderately decreases despite the stenosis.

The impaired cardiac performance becomes much more evident during exercise. Even at mild exercise $(I=0.4)$, stroke volume strongly decreases, and arterial pressure falls, too. On the contrary, heart rate exhibits a larger increase 
compared to the normal subject: in the stenosed patient, indeed, owing to hypotension, baroreceptors and the exercise-triggered mechanisms work in synergism on heart rate. The increase in heart rate warrants a certain augmentation in cardiac output, and so in blood flow to active muscles, too. However, this increase is much lower compared to the one in normal subjects, procuring serious limitation in the maximal oxygen uptake and exercise tolerance. In particular, model results reveal that when total oxygen body consumption exceeds 1.2-1.3 $\mathrm{l} / \mathrm{min}(I \geqslant 0.7)$, oxygen extraction becomes inadequate, owing to the poor cardiac output increase, and the patient must rely on anaerobic metabolism. Finally, model shows a strong increase in pulmonary venous pressure in stenosed patients performing exercise, revealing a tremendous damming of blood in the pulmonary vascular beds, and a high risk of pulmonary edema. Indeed, a large amount of blood volume is displaced from the systemic venous reserve by the regulatory mechanisms during exercise, and it is blocked by the failing left heart in the pulmonary circulation.

These model results agree with data reported in the clinical literature $[9,10]$.
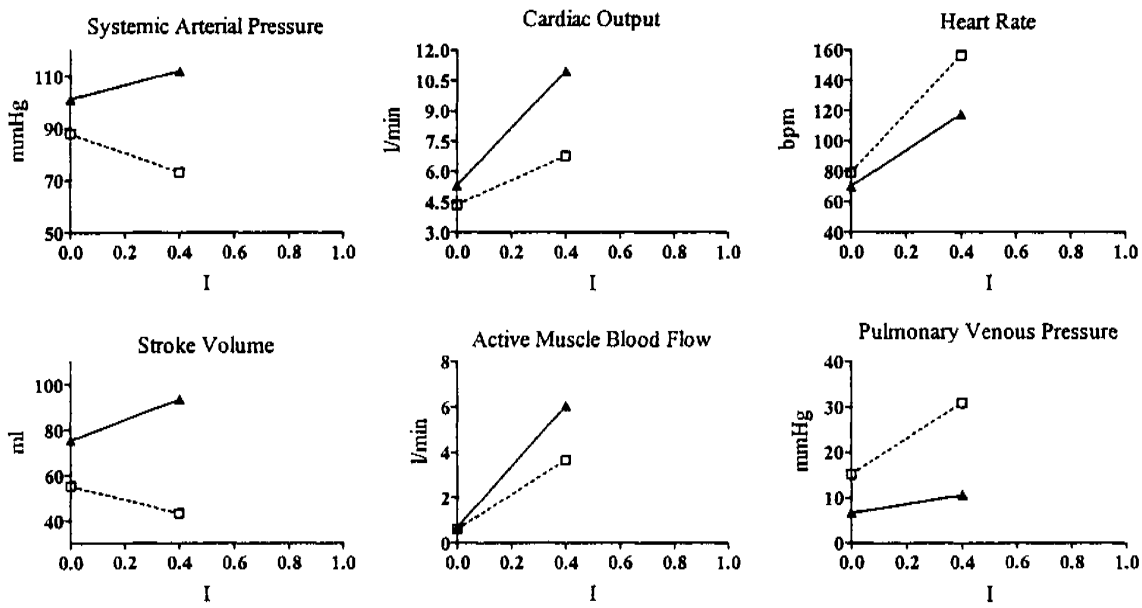

Figure 2: Mean values of the main hemodynamic quantities computed for a normal subject (A) and for a subject with moderate mitral stenosis $(\square)$, both at rest $(I=0)$ and during moderate exercise $(I=0.4)$. To simulate mitral stenosis, valve resistance between left atrium and left ventricle has been increased by 40 fold the basal value.

\section{Discussion}

Aim of the present work was to investigate the hemodynamic response to aerobic dynamic exercise by means of a computer model, both in healthy subjects and in subjects with heart insufficiency caused by mitral stenosis. The response to dynamic exercise, indeed, involves the superimposition of many concomitant 
factors, which interact in complex non-linear ways. The analysis of this problem is thus extremely difficult, while controversial assumptions can be found in the current literature in the field. We claim that a conclusive understanding of the experimental and clinical data can greatly benefit of a quantitative approach, based on mathematical modeling and computer simulation techniques.

The present model ascribes the cardiovascular response to dynamic exercise to the synergistic action of three primary mechanisms, which are directly triggered by the exercise: i) a reconfiguration of the autonomic neural (sympathetic and vagal) activity through a feedforward mechanism (usually named "the central command" in the physiological literature); ii) a local vasodilation in the active muscles, caused by the production of vasoactive metabolites and by tissue hypoxia; iii) the mechanical effect of the muscle and respiratory pumps on venous return. The previous mechanisms, in turn, interact with the secondary actions of a few additional reflex mechanisms (i.e., the baroreflex and the lung-stretch receptor reflex). The superimposition of these factors is able to explain the main aspects of cardiovascular physiology during exercise quite well, using parameter values for the single mechanisms which agree with those derived from physiological experiments. Hence, the model represents a convincing compendium of present knowledge on exercise cardiovascular control.

Among the three primary mechanisms mentioned above, model gives particular emphasis to the central command. Without this mechanism, in fact, exercise would result in a dramatic fall in systemic arterial pressure (secondary to the great metabolic vasodilation in the active muscle vascular bed) which can be only in part compensated by the arterial baroreflex response. In particular, the observation that systemic arterial pressure moderately increases during exercise (see Figure 1) can be explained only by a significant reconfiguration of sympathetic and vagal activity. There are several experimental evidences in the literature supporting the role of a central command in the response to aerobic exercise. These evidences come both from measurements in subjects attempting to make exercise efforts with paralyzed or inactivated muscles [11] and from stimulation of brain areas in animal experiments [12].

Simulations of patients with mitral stenosis emphasize two major aspects. First, cardiovascular quantities of a patient at rest are only moderately different compared with those of a healthy subject. Actually, stenosis of the mitral valve naturally worsens left ventricle filling at rest, which results in a decreased enddiastolic volume. As a consequence, one can observe a significant reduction in stroke volume (Figure 2). However, this condition is masked by the action of regulatory mechanisms, which increase heart rate and constrict arterioles and veins, thus restoring almost normal levels of cardiac output and mean systemic arterial pressure, while peripheral blood flow exhibits only mild reductions. The pathological status, however, which is hidden at rest, becomes dramatically evident even at moderate exercise levels. In this condition, in fact, two negative phenomena occur. First, pressure drop at the mitral valve increases, as a consequence of the increased cardiac output, further limiting the filling of the ventricle. Second, the regulatory mechanisms have already exploited part of their 
compensatory reserve, and may saturate. The consequences are the fall in stroke volume and mean systemic arterial pressure, the disproportionate increase in heart rate and, finally, a limitation in oxygen delivery and exercise tolerance. All these aspects, predicted by model simulations, agree with typical scenarios observed in patients with mitral stenosis performing exercise tests [5].

The previous results confirm the importance of testing a patient during exercise to analyze his/her cardiovascular reserve and to reveal the grade of cardiac insufficiency. In perspective, the present model may be a potential support for clinical reasoning, to design quantitative exams of cardiac performance, based on hemodynamic changes between rest and exercise.

\section{References}

[1] Ursino, M. Interaction between carotid baroregulation and the pulsating heart: a mathematical model. Am. J. Physiol., 275 (Heart Circ. Physiol. 44), pp. H1733-H1747, 1998.

[2] Ursino, M. \& Magosso, E. Acute cardiovascular response to isocapnic hypoxia. I. A mathematical model. Am. J. Physiol. Heart Circ. Physiol., 279, pp. H149-H165, 2000.

[3] Ursino, M. \& Magosso, E. Acute cardiovascular response to isocapnic hypoxia. II. Model validation. Am. J. Physiol. Heart Circ. Physiol., 279, pp. H166-H175, 2000.

[4] Magosso, E. \& Ursino, M. A mathematical model of $\mathrm{CO} 2$ effect on cardiovascular regulation. Am. J. Physiol. Heart Circ. Physiol., 281, pp. H2036-H2052, 2001.

[5] Laughlin, M.H. Cardiovascular response to exercise, Am. J. Physiol. 277 (Adv. Physiol. Educ. 22), pp. H1733-H1747, 1998.

[6] Rowell, L.B. Human Cardiovascular Control, Oxford University Press: New York, 1993.

[7] Pedley, T.J. The Fluid Mechanics of Large Blood Vessels, Cambridge University Press: Cambridge, 1980.

[8] Pawelczyk, J.A., Hanel, B., Pawelczyk, R.A., Warberg, J. \& Secher, N.H. Leg vasoconstriction during dynamic exercise with reduced cardiac output. J. Appl. Physiol., 73(5), pp. 1838-1846, 1992.

[9] Lange, R.A., Moore, D.M., Cigarro, R.G. \& Hillis, L.D. Use of pulmonary capillary wedge pressure to assess severity of mitral stenosis: is true left atrial pressure needed in this condition? J. Am. Coll. Card., 13, pp. 825-831, 1989.

[10] Guyton, A.C. Textbook of Medical Physiology, W.B. Saunders Company: Philadelphia, 1986

[11] Secher, N.H. Heart rate at the onset of static exercise in man with partial neuromuscular blockade. J. Physiol., 368, pp. 481-490, 1985.

[12] Elridge, F.L., Millhorn, D.E., Kiley, J.P. \& Waldrop, T.G. Stimulation by central command of locomotion, respiration and circulation during exercise. Respir. Physiol., 59, pp. 313-337, 1985. 